\title{
ANALYSIS OF POLARIZATION ORIENTATION ANGLE BASED ON SCATTERING MECHANISMS
}

\author{
Jong-Sen Lee ${ }^{1,2}$, Thomas L. Ainsworth ${ }^{1}$, and Yanting Wang $^{1}$ \\ ${ }^{1}$ Remote Sensing Division,Naval Research Laboratory, Washington DC, USA \\ ${ }^{2}$ Computation Physics Inc., Springfield, Virginia, USA \\ jong_sen_lee@yahoo.com
}

\begin{abstract}
The complexity of polarization orientation angle induced by scatterings from buildings and distributed media will be investigated. We will look into 1) the estimation algorithm, 2) The double bounce scattering from building walls not aligned in the along-track direction and 3) the orientation angle associated with very high-resolution PolSAR images with decimeter resolution.
\end{abstract}

Index Terms - Polarimetric SAR, scattering modeling, Orientation angle estimation

\section{INTRODUCTION}

Polarization orientation angle (POA) is the angle of rotation about the line of sight. For reflection symmetrical media, such as horizontal surface, the orientation angle is zero. For polarimetric SAR (PolSAR) data from rugged terrain areas and for buildings in urban areas, POA is shifted from zero. POA shifts are induced by surfaces with nonzero azimuth slopes [1 - 3], and also by manmade targets, such as buildings that are not aligned in the along-track direction and bridges with tilted linear structures [4,5]. For distributed media, in general, the higher is the radar frequency; the lower is the orientation angle sensitivity to the surface slopes. It can be more accurately estimated in Lband and P-band PolSAR data than in C-band and X-band for surface slopes. The induced polarization orientation angle $\boldsymbol{\theta}$ is related to the azimuth and range slopes by the following equation $[1,2]$,

$$
\tan \theta=\frac{\tan \omega}{-\tan \gamma \cos \phi+\sin \phi}
$$

where $\tan \omega$ is the azimuth slope, $\tan \gamma$ is the slope in the ground range direction, and $\phi$ is the radar look angle. The orientation angle can be easily computed by the angle of the correlation between LL and RR circular polarizations,

$$
\begin{aligned}
\tan (-4 \eta) & =\frac{-4 \operatorname{Re}\left(<\left(S_{H H}-S_{V V}\right) S_{H V}^{*}>\right)}{-<\left|S_{H H}-S_{V V}\right|^{2}>+4<\left|S_{H V}\right|^{2}>} \\
\theta & =\left\{\begin{array}{ccc}
\eta, & \text { if } & \eta \leq \pi / 4 \\
\eta-\pi / 2, & \text { if } & \eta>\pi / 4
\end{array}\right.
\end{aligned}
$$

In (2), $\operatorname{Re}(\mathbf{A})$ denotes the real part of $A$ and the factor of 4 in $4 \boldsymbol{\theta}$ limits the range of $\theta$ to $[-\pi / 4, \pi / 4]$.

\section{PROBLEMS ASSOCIATED WITH POA ESTIMATION}

The following issues were often raised on POA estimation: 1) POA is phase wrapped just like the interferometry wrapped phase, but unlike interferometry, no fringes exist to aid phase unwrapping. 2) Backscatterings from urban environment are, in general, more complex. For example, for backscatterings from building not aligned in the along-track direction, there exist surface scatterings from the roof and wall, double bounce from the wall to the rough ground or surrounding landscape and back to radar, which is not the idealized double bounce scattering, where POA is zero. In addition, triple bounce scattering from wall to ground to wall and back to radar could play a role in POA. The radar returns are the combination of all three scattering mechanisms, and their orientation angle depends on the dominant scattering mechanism. 3) High resolution PolSAR images may add to the understanding of scattering mechanisms for POA from buildings and from distributed targets. However, high POA variations are expected.

In this paper, we will investigate the following:

1) The POA estimation algorithm including the phase unwrapping issues for both distributed media and urban areas will be further studied based on scattering mechanisms.

2) The scattering from building walls not aligned in the along-track direction can also introduce double bounce scattering due to the rough surface of surrounding landscape. We shall call this "tilted double bounce scattering". We will investigate the induced orientation angle from the tilted double bounce scattering and its relation to the tilted angle. 
3) Very high-resolution PolSAR images with decimeter resolution present the advantage to understand the basic scattering mechanisms. This is because the scattering in each pixel is likely associated with a single dominant scattering mechanism. For forested areas, the randomness in POA distribution may offer an advantage in vegetation discrimination.

\section{ORIENTATION EFFECT OF BUILDINGS}

Using E-SAR L-band PolSAR data of Dresden, Germany, we test the POA extraction algorithm of (2) and (3). The Freeman/Durden decomposition image is shown in Fig. 1A. The SAR look direction is from left to right of the image. The POA image is shown in $1 \mathrm{~B}$ with color coded by $1 \mathrm{C}$. We observe that double bounce pixels shown in red in $1 \mathrm{~A}$ have their POA close to zero in (B), whereas buildings not aligned in the along-track direction has higher POA as shown with different color. It is apparent the POA range of $[-\pi / 4, \pi / 4]$ causes phase wrapping. To consistently unwrap the phase, we need to look into the scattering mechanism, such as the tilted double bounce scattering.

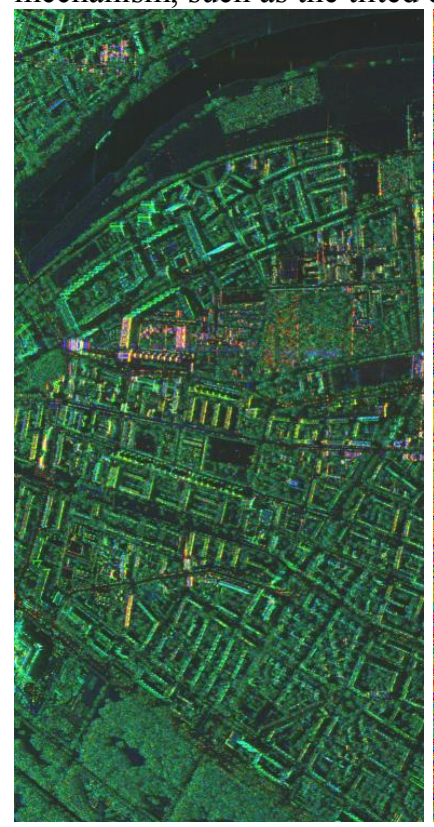

(A) Freeman decomposition

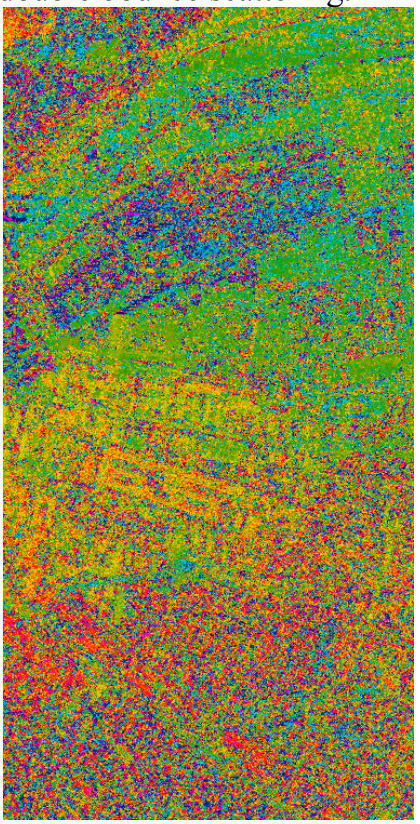

(B) POA according to $(C)$

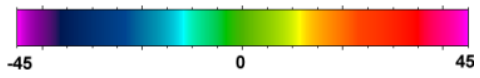

(C) Color label for POA

Fig. 1 E-SAR L-band PolSAR data of Dresden is used for illustration for POA estimation. The image is displayed with Freeman/Durden decomposition in (A), and the orientation angle is shown in (B) with color coded by $(C)$. We observe that double bounce pixels shown in red in $(A)$ have their orientation angle close to zero in (B), whereas buildings not aligned in the along-track direction has different POA as shown with different color. It is apparent the POA range of $[-\pi / 4, \pi / 4]$ cause phase wrapping.

The geometric orientation of buildings affects their POA. Kimura et.al [4, 5] have shown that the same estimation algorithm (2) and (3) can be applied to obtain the POA that to a certain degree correspond to the building orientation. Assuming the building wall is aligned at an angle $\alpha$ with the along-track direction as shown in Fig. 2. Kimura [4] extended (1) to obtain POA for building walls geometrically, which makes that corresponding look angle for this case to be $(\boldsymbol{\pi} / \mathbf{2}-\boldsymbol{\phi})$. Since most building walls are vertical, the range slope becomes zero. With these two assumptions, (1) becomes

$$
\tan \theta=\frac{-\tan \alpha}{\cos \phi}
$$

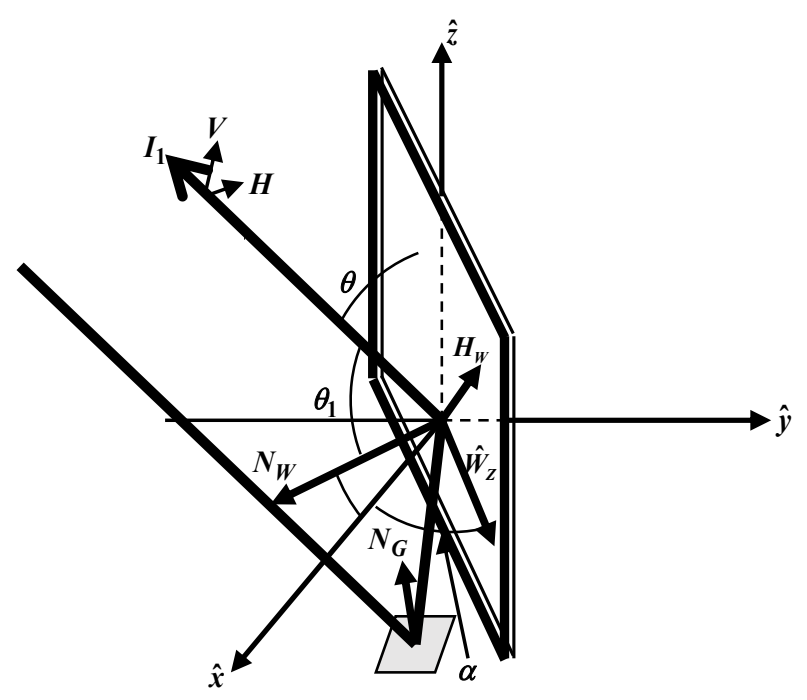

Fig. 2 Schematic scattering diagram of a building wall. The incidence plane is in the $y-z$ plane. The $x$-axis is in the along-track direction. This figure shows the configuration of the tilted double bounce backscattering.

Recently high-resolution SAR systems have been developed that can achieve decimeter resolution with low noise floor and good polarization channel separation. DLR F-SAR is such a system. With 0.25 meter resolution in $\mathrm{X}$ band and .65 meter in S-band, F-SAR PolSAR data provide the advantage to study POA induced by buildings, because it is likely that each resolution cell is dominated by a single scatterer. On the other hand, the POA may have high variation that make analysis more difficult, especially in distributed targets. We tested F-SAR X-band PolSAR data and the result is shown in Fig. 4. The conventional Pauli vector color coding is shown in $4 \mathrm{~A}$, its abs(POS) is color coded in $4 \mathrm{~B}$. The color pattern is from blue to green to red to yellow. Figure $4 \mathrm{~B}$ exhibits high variation in POA. To visualize the POA estimation from buildings, we overlay the 
color of $4 \mathrm{~B}$ on the span of $4 \mathrm{~A}$, and the result in $4 \mathrm{C}$ clearly shows the POA from buildings. We also note the high variation in POA surface scattering areas and in volume scattering areas. The POA distribution in forested area may offer an advantage in vegetation discrimination.

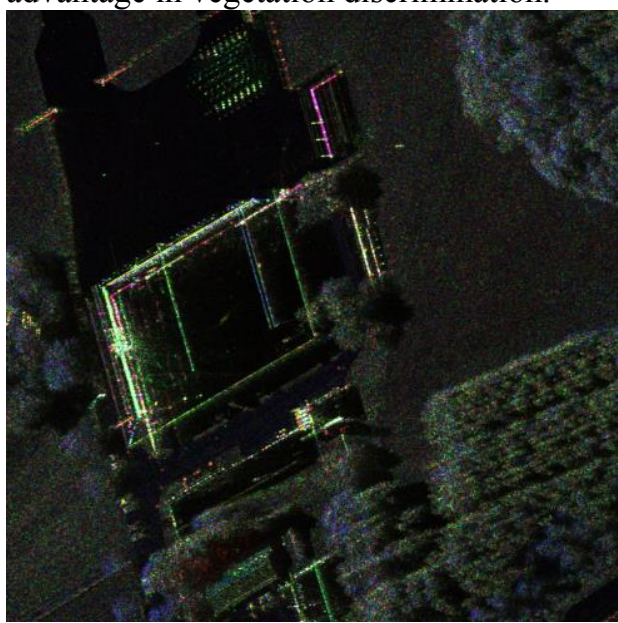

(A) Pauli colored image

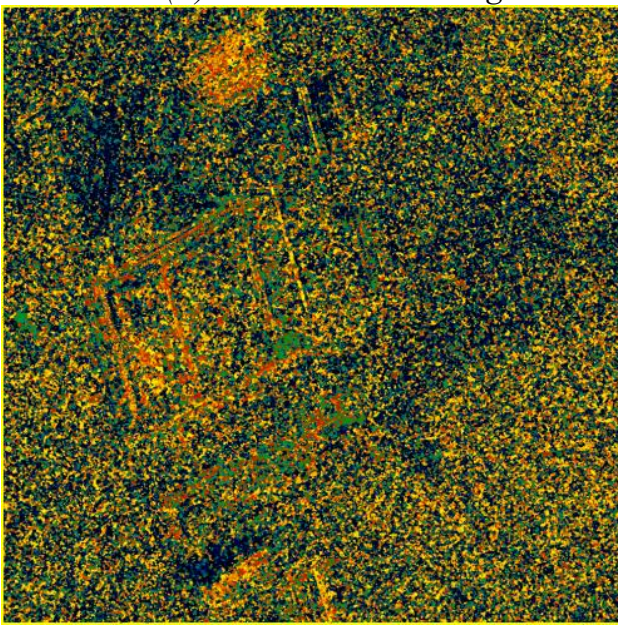

(B) POA in absolute value

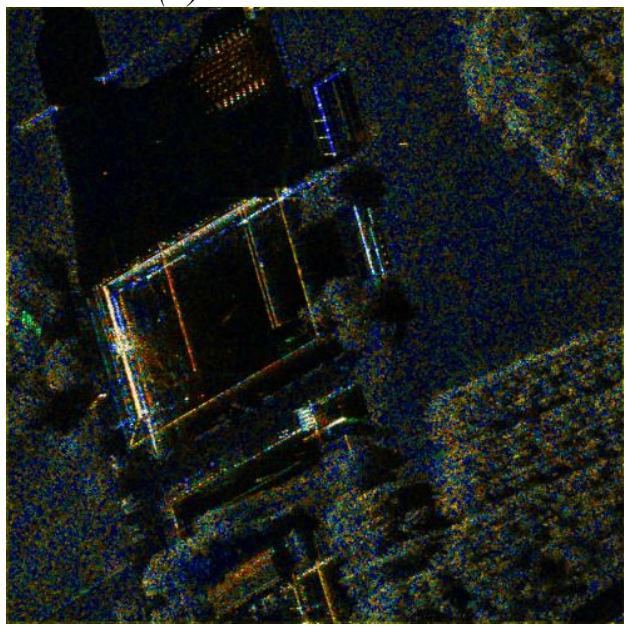

(C) Overlay (B) with the span

Fig. 4 POA estimation from high resolution F-SAR $X$ band PolSAR data. The conventional Pauli vector color coding is shown in (A), its abs(POS) is color coded in $(B)$. The color pattern is from blue to green to red to yellow. To enhance the POA of buildings and hard targets, we overlay the color of $(B)$ with the span of the image. The result is shown in $(C)$.

\section{REMARKS}

The geometric POA equation (6) were derived from surface scattering rather than for urban area, and it may not be directly applied to scattering from building walls. In reality, wall surface is rather smooth on the scale of radar wavelength, backscattering power from wall surface is generally negligible. The dominant backscattering most likely comes from tilted double bounce from radar to wall to the ground and back to radar. This is because, just like the typical double bounce scattering, there are multiple paths that have identical distance, accumulating higher return. Consequently, further research is required.

Triple bounce scattering from radar to wall to ground and back to wall and to radar has only a single path. It may not produce significant returns because of the single path. Of course, exceptions exist. For example, scattering from bridge structures and water surface.

\section{SUMMARY}

The complexity of polarization orientation angle induced by scatterings from buildings and distributed media has been shown. Currently, we are working on unwrapping of orientation angle and the tilted double bounce scattering to obtain the relation between POA derived from PolSAR data and the wall angle $\alpha$ from radar geometry.

\section{REFERENCES}

[1] J.S. Lee, D.L. Schuler and T.L. Ainsworth, "Polarimetric SAR data compensation for terrain azimuth slope variation," IEEE Trans. on Geoscience and Remote Sensing, vol. 38, no. 5, pp. 2153-2163, 2000.

[2] J.S. Lee, D.L. Schuler, T.L. Ainsworth, E. Krogager, D. Kasilingam, and W.-M. Boerner, "On the estimation of radar polarization orientation shifts induced by terrain slopes," IEEE Trans. on Geoscience and Remote Sensing, vol. 40, no.1, pp. 30-41, 2002.

[3] F. Xu, and Y.-Q. Jin, "Deorientation theory of polarimetric scattering targets and application to terrain surface classification," IEEE Trans. on Geoscience and Remote Sensing, vol.43, no.10, pp. 2351-2364, 2005. 
[4] H. Kimura, K.P. Papathanassiou and I. Hajnsek, "Polarization Orientation Effect in Urban Areas on PolSAR Data," Proceedings of IGARSS 2005, pp. 48634867, Seoul, July 2005.

[5] K. Irebe and M. Sato, "Analysis of Polarization Orientation Angle Shifts by Artifical Structures," IEEE Trans. on Geoscience and Remote Sensing, vol.45, no.11, pp. 3417$3425,2007$. 